\title{
Spatial observations by the CUTLASS coherent scatter radar of ionospheric modification by high power radio waves
}

\author{
G. E. Bond ${ }^{1}$, T. R. Robinson ${ }^{1}$, P. Eglitis ${ }^{1}$, D. M. Wright ${ }^{1}$, A. J. Stocker ${ }^{1}$, M. T. Rietveld ${ }^{2}$ and T. B. Jones ${ }^{1}$ \\ ${ }^{1}$ Department of Physics and Astronomy, Leicester University, University Road, Leicester, LE1 7RH, UK \\ ${ }^{2}$ EISCAT Scientific Association, Ramfjordmoen, N-9027 Ramfjorbotn, Norway
}

Received: 5 February 1997 / Revised: 12 May 1997 / Accepted: 14 May 1997

\begin{abstract}
Results are presented from an experimental campaign in April 1996, in which the new CUTLASS (Co-operative UK twin-located Auroral Sounding System) coherent scatter radar was employed to observe artificial field aligned irregularities (FAI) generated by the EISCAT (European Incoherent SCATter) heating facility at Tromsø, Norway. The distribution of backscatter intensity from within the heated region has been investigated both in azimuth and range with the Finland component of CUTLASS, and the first observations of artificial irregularities by the Iceland radar are also presented. The heated region has been measured to extend over a horizontal distance of $170 \pm 50 \mathrm{~km}$, which by comparison with a model of the heater beam pattern corresponds to a threshold electric field for FAI of between 0.1 and $0.01 \mathrm{~V} / \mathrm{m}$. Differences between fieldaligned and vertical propagation heating are also presented.
\end{abstract}

\section{Introduction}

When high power radio waves, operating in ordinary polarisation, are beamed vertically and at an angle within a few tens of degrees of the geomagnetic field, they strongly excite plasma density irregularities in regions where the wave frequency is close to the local upper-hybrid frequency (Stubbe et al., 1982; Robinson, 1989 and references therein). These plasma irregularities are highly elongated in the direction of the geomagnetic field, as a consequence of the highly anisotropic nature of electron transport in the ionosphere. Small-scale fieldaligned irregularities (FAI) with scale sizes of a few metres to tens of metres across the geomagnetic field coherently backscatter radar signals when the radar beams are directly orthogonal to the geomagnetic field.

Correspondence to: G. E. Bond
In early ionospheric modification experiments using the high power radio transmitter at Boulder, Colorado, Thome and Blood (1974) detected strong coherent backscatter at both VHF and HF frequencies. These experiments were followed by a number of Russian investigations (e.g. Belenov et al., 1977; Korovin et al., 1982). More recently, Hedberg et al. (1983, 1986) and Hanuise et al. (1986) have detected HF backscatter from artificial FAI in the auroral F-region, produced by the EISCAT high power facility at Tromsø, Norway (Rietveld et al. 1993). In addition, Hibberd et al. (1983) and Djuth et al. (1985) have detected VHF backscatter from E-region FAI generated by the Tromsø heater. Coster et al. (1985) utilised a $50 \mathrm{MHz}$ radar to measure the growth and decay times of irregularities in both the E- and F-regions, produced by the heating facility at Arecibo, Puerto Rico.

CUTLASS (Co-operative UK Twin Located Auroral Sounding System) is a new HF coherent scatter system with identical radars in Finland and Iceland (Greenwald et al. 1995). The system is ideally positioned to monitor RF modification experiments performed with the EISCAT heater. The CUTLASS Finland radar lies approximately $1000 \mathrm{~km}$ to the south of Tromsø, and from this position the radar can take measurements over a horizontal cross section in the F-region above the heater. The arrangement also facilitates simultaneous observations to be made with the EISCAT incoherent scatter radars (Rishbeth and Williams, 1985). Both CUTLASS sites make use of 16 antennas in their main arrays, which are each connected to separate $600 \mathrm{~W}$ RF power amplifiers. The beam formed by the 16 antennas is $3.24^{\circ}$ wide, and can be steered in any combination of 16 directions. CUTLASS transmits $300 \mu$ s pulses in standard mode, giving a range resolution of $45 \mathrm{~km}$. This resolution has recently been improved to $15 \mathrm{~km}$ by the use of $100 \mu$ s pulses.

In this study we present CUTLASS observations of FAI produced by the EISCAT heater, during an experimental campaign in April 1996. CUTLASS was configured in a high resolution mode to map the heated 


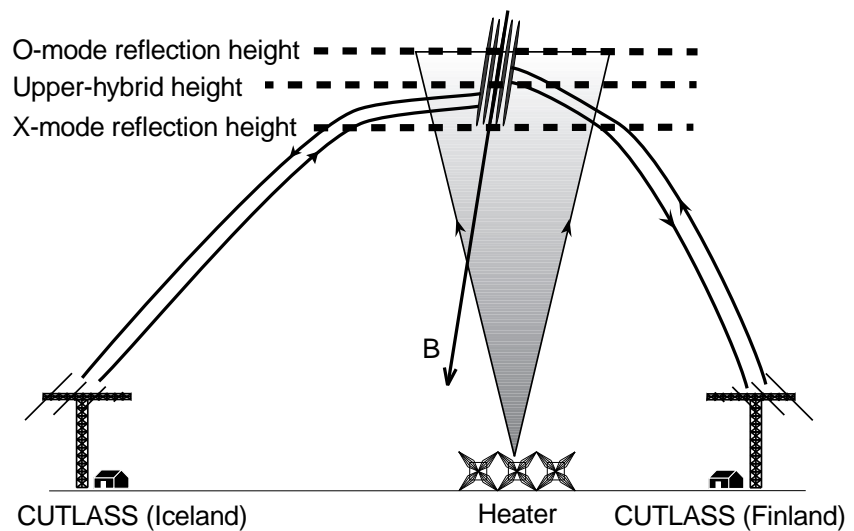

Fig. 1. A schematic illustrating the relative beam geometry of the EISCAT heater and the CUTLASS radars

region in greater detail than has been possible in previous radar investigations of FAI. We indicate the distance over which FAI are observed, both in range and azimuth, and present measurements of the spatial distribution of backscatter power from the FAI.

\section{Experimental arrangement}

The arrangement of diagnostics and the heater for modification experiments carried out in April 1996 is depicted schematically in Fig. 1. The heater operated on frequencies between 3.9000 and $4.9128 \mathrm{MHz}$, with 6 of the 12 available heater transmitters, along with half the dipole antennas. The six transmitters operated at $90 \mathrm{~kW}$ each, and the gain of the array was $21 \mathrm{~dB}$, compared to
$24 \mathrm{~dB}$ when the full array is in use. This gave a heater ERP of just under $70 \mathrm{MW}$, neglecting losses. Both the Iceland and Finland components of the CUTLASS system were operational, and transmitted frequencies between 8 and $20 \mathrm{MHz}$, along up to five adjacent beam directions positioned to intercept the heated region over Tromsø. The Finland CUTLASS radar was utilised in a high resolution mode which produced a temporal resolution as fine as $1 \mathrm{~s}$ per beam compared to the usual $7 \mathrm{~s}$, and a nominal range resolution of $15 \mathrm{~km}$ compared to the standard $45 \mathrm{~km}$ resolution. For the CUTLASS high resolution scan mode, the range cells are $15 \mathrm{~km}$ long and approximately $45 \mathrm{~km}$ across the beams at Troms $ø$. The length of a cell along a beam is determined by the length of the pulse utilised by the radar, and the width of a cell across the beams is determined by the beam width of the radar.

The EISCAT UHF radar and a low power diagnostic system were also operational during the campaign, but those results are not presented here. The results of simultaneous observations with multiple diagnostics from an earlier campaign are presented in Robinson et al. (1997).

\section{Observations}

\subsection{CUTLASS observations of the horizontal extent of the heated region}

The map in Fig. 2 illustrates the relative positions of the two CUTLASS radars in Pykkvibær, Iceland and Hankasalmi, Finland. The region overlooked by the two radars includes the EISCAT heater and incoherent

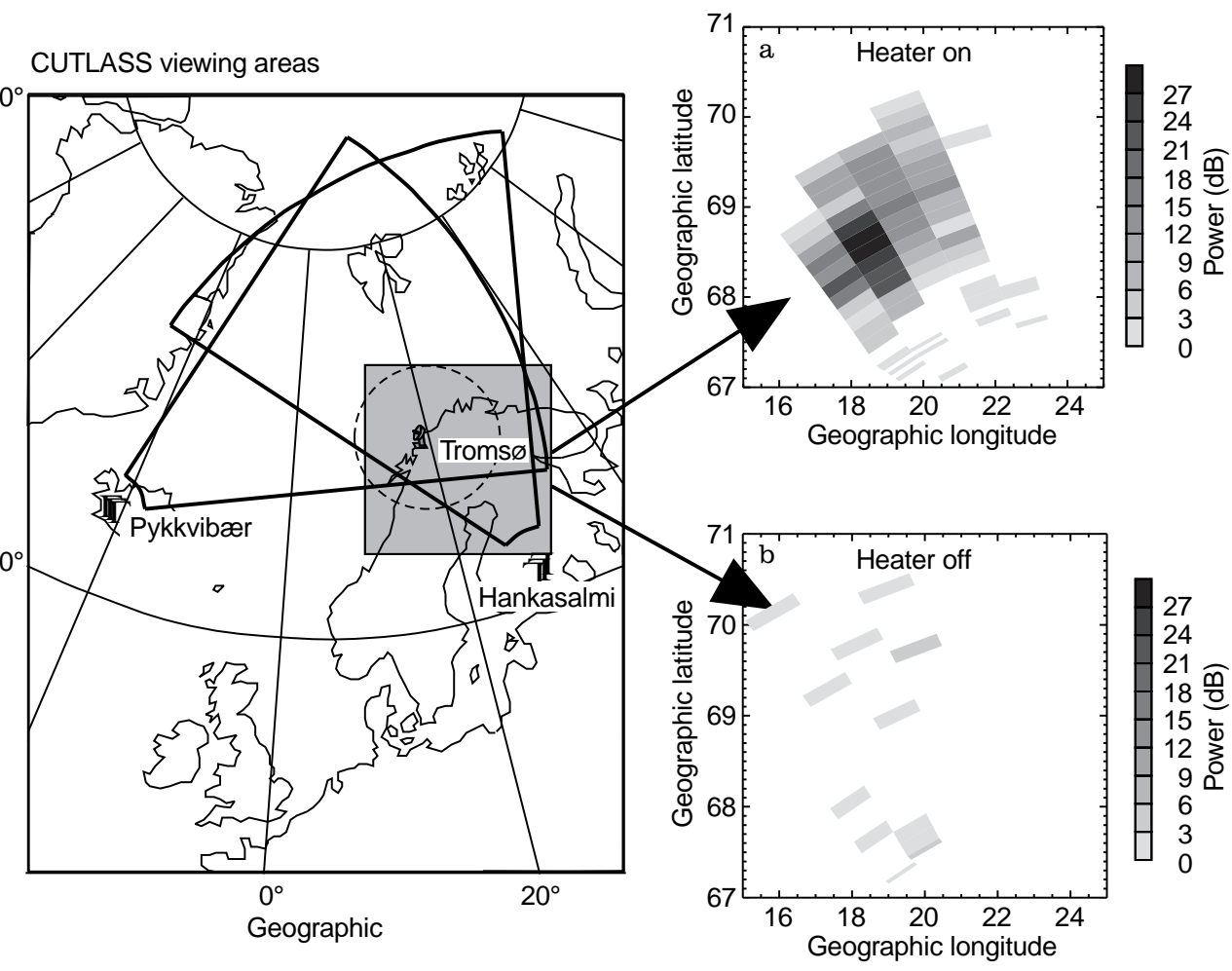

Fig. 2a, b. The map on the left depicts the locations and fields-ofview of the CUTLASS and EISCAT radars. The shaded region indicates the area covered by the spatial plots presented in $\mathbf{a}$ and $\mathbf{b}$. a Returned power from the CUTLASS Finland radar on 26 April 1996, during a heater-on period. b Data taken 4 min later during a heater off period 
scatter radar at Tromsø. The data in Fig. 2a is for $1200^{33}$ UT on 26 April 1996. The CUTLASS Finland radar was operating at $15 \mathrm{MHz}$ along beams 3-7 and the heater pointed vertically, transmitting at $4.04 \mathrm{MHz}$ in $\mathrm{O}-$ mode polarisation. The scatter depicted in panel $a$ provides evidence of artificial FAIs. This is confirmed in Fig. 2b, which illustrates the absence of backscatter in the CUTLASS field of view during the following heateroff period. The data in Fig. 2 indicate that the region where FAIs were excited had a horizontal extent of around $200 \mathrm{~km}$. This representation is slightly inaccurate, as the width of the radar beam and the length of the pulse are convolved with the true distribution of FAI in the heated region. In order to find the true horizontal extent of the heated region it is necessary to deconvolve the shape of the pulse (or beam) from the data produced by CUTLASS. One way of achieving this is to fit analytical Gaussian profiles to the data. In addition to providing a deconvolution method, this technique also indicates how well the returned power from the heated region conforms to a Gaussian distribution. A Gaussian profile is chosen because it represents a simple, wellbehaved function which has a shape determined only by its width and peak amplitude.

Figure $3 \mathrm{a}$ illustrates the returned power from CUTLASS Finland along beam 5, averaged over the 3 min Omode heating period beginning at 1224 UT on 26 April 1996. The dotted line represents the data from CUTLASS and the solid line represents a Gaussian function fitted to the data, of the form

$k_{\text {data }} \mathrm{e}^{-x^{2} / 2 \sigma_{\text {data }}^{2}}$,

where $k_{\text {data }}$ is the maximum height of the Gaussian and $2 \sigma_{\text {data }}$ is the full width. The fit is achieved by matching the area under the dotted line to the area under the Gaussian, and then changing the width until a leastsquares-fit is found. Figure $3 \mathrm{~b}$ presents the power output produced when a square-wave test input is fed into a CUTLASS receiver. The pulse length is converted from the temporal to the spatial domain by multiplying the width in seconds by half the free-space speed of light. The dotted line represents the data from the receiver and the solid line represents the best-fit Gaussian to the data, which has the form

$k_{\text {pulse }} \mathrm{e}^{-x^{2} / 2 \sigma_{\text {pulse }}^{2}}$,

where $k_{\text {pulse }}$ is the height of the profile and $2 \sigma_{\text {pulse }}$ is the full width. A Gaussian representing the distribution of FAI within the heated volume can be found by deconvolving the Gaussian representing the pulse from the Gaussian representing the data. This deconvolution is achieved by first finding the Fourier $(\omega)$ transforms of the curves in expressions 1 and 2. These transforms have the form

$$
\begin{aligned}
& \sqrt{2 \pi} k_{\text {data }} \sigma_{\text {data }} \mathrm{e}^{-2 \sigma_{\text {data }}^{2} \omega^{2} / 4} \\
& \sqrt{2 \pi} k_{\text {pulse }} \sigma_{\text {pulse }} \mathrm{e}^{-2 \sigma_{\text {pulse }}^{2} \omega^{2} / 4}
\end{aligned}
$$

Dividing Eq. (3a) by (3b) gives the Fourier transform of the deconvolved Gaussian which represents the distri-
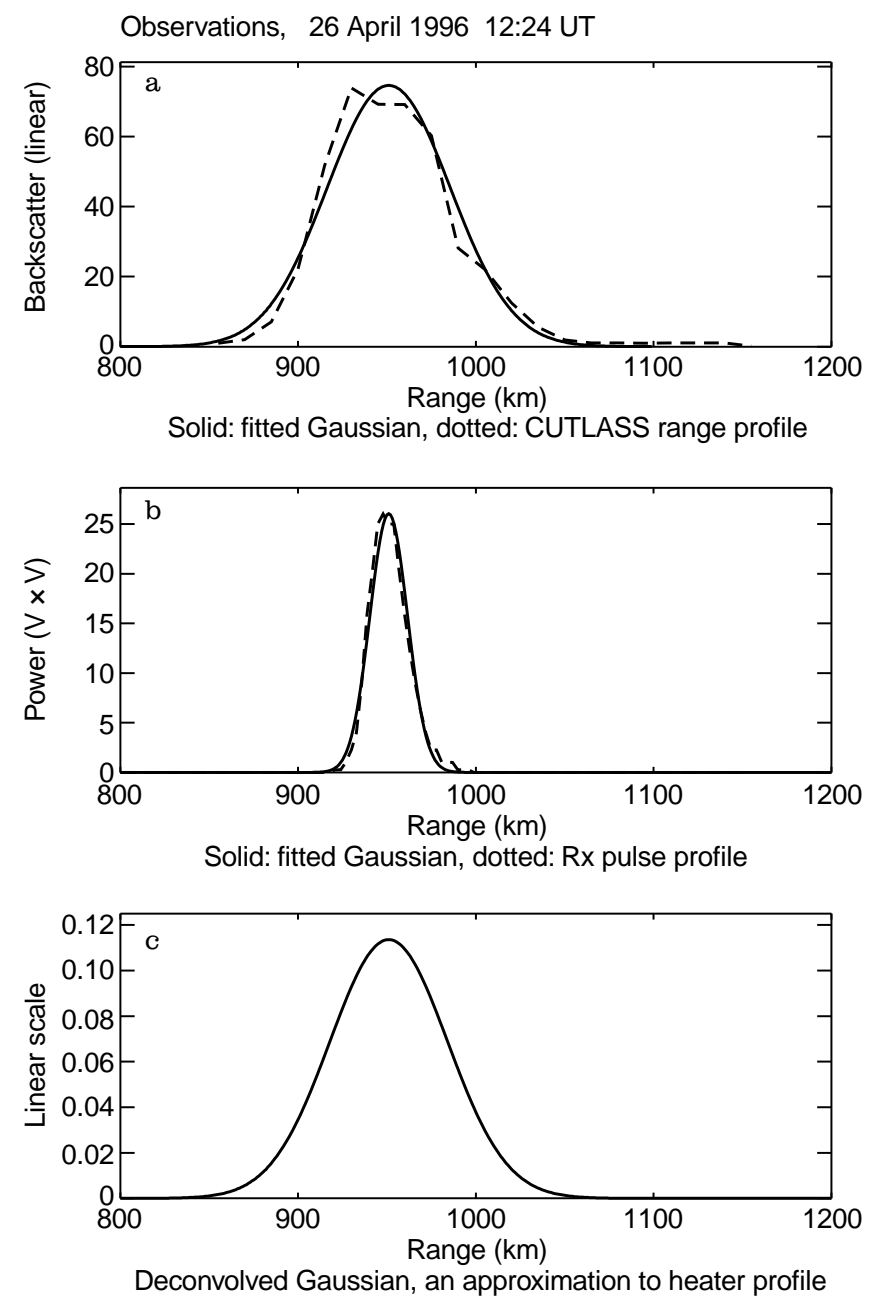

Fig. 3a-c. Observations for 1224 UT on 26 April 1996. a The dotted line represents backscatter power from CUTLASS Finland along beam 5. The solid line is a Gaussian function fitted to the data. $\mathbf{b}$ The dotted line represents the power produced by a pulse fed into a CUTLASS receiver. The solid line is a Gaussian fitted to the profile. c An analytical profile produced by deconvolving the Gaussian fitted to the pulse profile from the curve fitted to the backscatter data

bution of FAI. After inverse transforming the resulting deconvolved profile has the form.

$\mathrm{e}^{-x^{2} / 2\left(\sigma_{\text {data }}^{2}-\sigma_{\text {pulse }}^{2}\right)}$.

This profile is plotted in Fig. 3c, and the full width is $2 \sqrt{\sigma_{\text {data }}^{2}-\sigma_{\text {pulse }}^{2}}$.

Figure 4 presents data from the same time as depicted in Fig. 3. The dotted line in the top panel represents the returned power from CUTLASS Finland across five beam directions, at a range corresponding to the position of the heater. The solid line is a best-fit Gaussian to the data, of the form illustrated in expression 1. The dotted line in Fig. 4b depicts a modelled profile for beam 5 of the CUTLASS radar (the profiles for the other beams utilised in this experiment are very similar). The solid line represents a best-fit Gaussian to the data, with the same form as expression 2. The bottom panel of Fig. 4 illustrates the result of analyt- 
Observations, 26 April 1996 12:24 UT

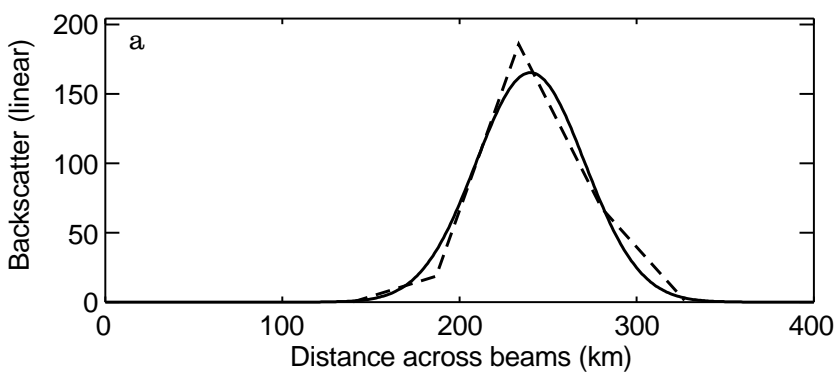

Solid: fitted Gaussian, dotted: CUTLASS beam profile

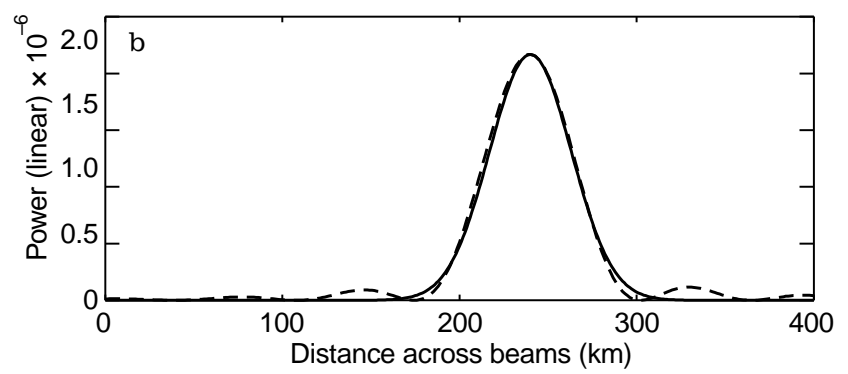

Solid: fitted Gaussian, dotted: beam profile

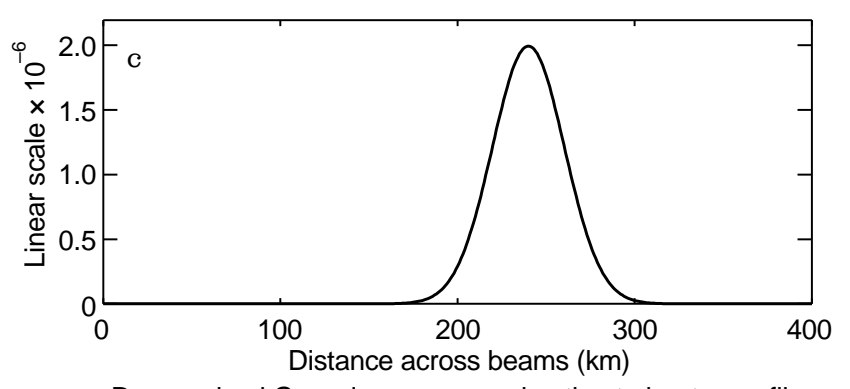

Deconvolved Gaussian, an approximation to heater profile

Fig. 4. Observations for 1224 UT on 26 April 1996. a The dotted line represents backscatter power from CUTLASS Finland across beams 3-7 at a range gate over Tromsø. The solid line is a Gaussian function fitted to the data. $\mathbf{b}$ The dotted line represents a modelled beam profile for the CUTLASS antenna system. The solid line is a Gaussian fitted to the beam profile. $\mathbf{c}$ An analytical profile produced by deconvolving the Gaussian fitted to the beam profile from the curve fitted to the backscatter data

ically deconvolving the Gaussian fitted to the beam shape from the Gaussian fitted to the CUTLASS data. The profile is a representation of the extent of the heated region across the beams; and has the same form as expression 4.

In order to interpret spatial observations of the heated volume it is necessary to model the beam pattern of the heater. During the April 1996 campaign, only 6 of the 12 transmitters were available at the heater site, along with half the dipole antennas of array 2 . Whilst the full number of antennas were available in the eastwest direction, only half the usual number in the northsouth direction were present. Therefore, the beam pattern was modelled to take account of the northsouth, east-west asymmetry. The heater beam pattern was calculated from the theoretical beam pattern of the heater antenna array. In addition, ray tracing calculations were carried out utilising a realistic electron

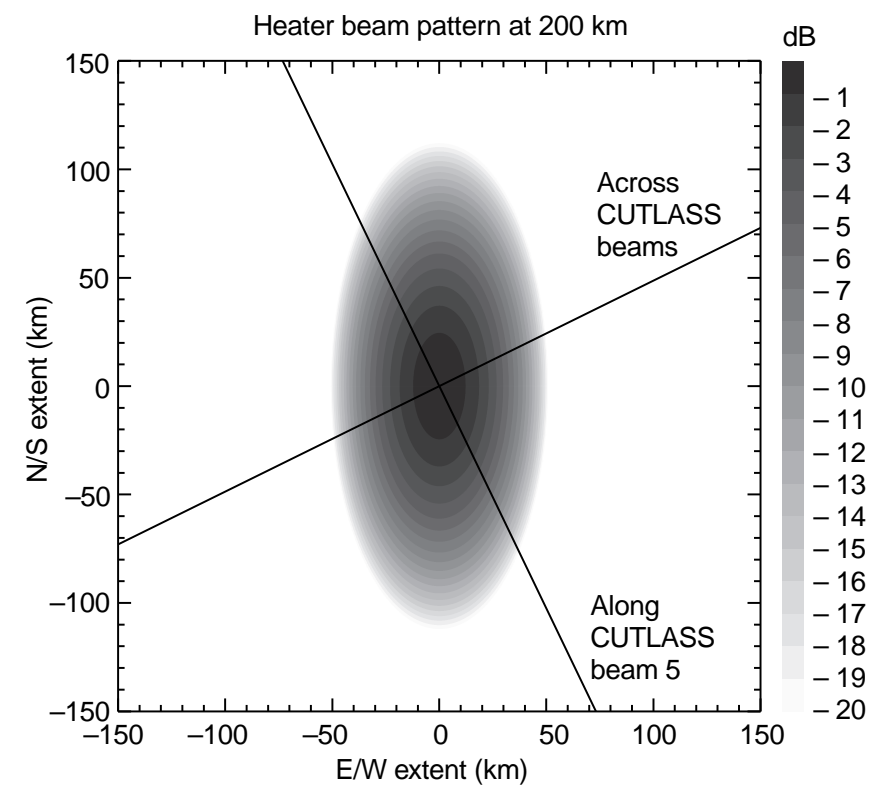

Fig. 5. A contour plot illustrating the power distribution of the heater beam at an altitude of $200 \mathrm{~km}$. The shading presents the power in $\mathrm{dB}$ below the power at the centre of the beam. The straight lines indicate the directions along and normal to CUTLASS Finland beam 5

density profile which indicated that spreading of the heater rays near the reflection point was less than $5 \mathrm{~km}$. Figure 5 presents the contours at a height of $200 \mathrm{~km}$ for $\mathrm{O}$-mode propagation at $4.04 \mathrm{MHz}$. The contours are expressed in $\mathrm{dB}$ below the power at the centre of the beam. Each contour is an ellipse, with the major axis determined by the beam pattern in the north-south direction, and the minor axis by the beam pattern in the east-west direction. The direction of CUTLASS beam 5 is marked in Fig. 5, along with the direction across the CUTLASS beams. By calculating where these two lines cross the contours it is possible to build up a picture of

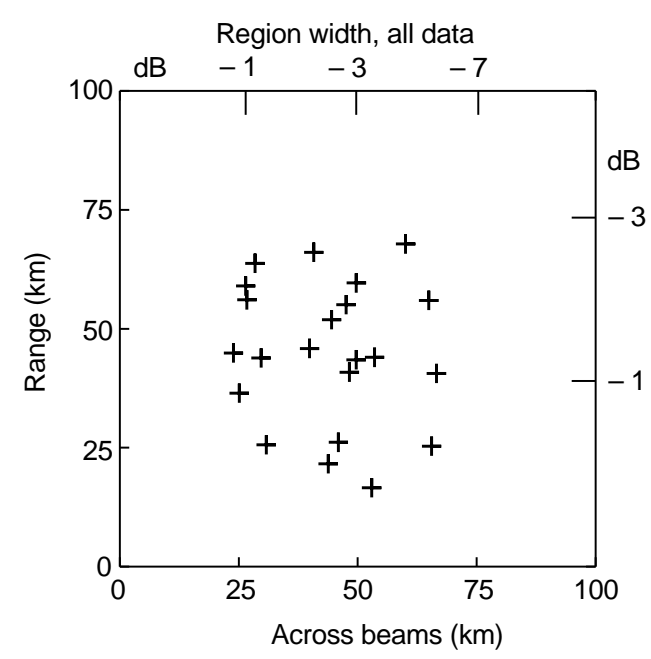

Fig. 6. Observations for 22-26 April 1996. The scatter plot represents the width of the heated region along CUTLASS beam 5 against the width across CUTLASS beams 3-7. The dB scale indicates the level of power in $\mathrm{dB}$ down from the centre of the heater beam 
the amount of heater power radiated into different positions in the CUTLASS field of view.

Observations covering the whole campaign are depicted in Fig. 6. The data is from CUTLASS Finland, for times when the radar was operating in a beam 3-7 scan mode. Each point in the scatter plot represents data averaged over an O-mode heater period (data from Xmode heater periods did not produce any significant scatter). The y-coordinate of each point represents the deconvolved width of the heated region along the radar range. This is determined by taking measurements of CUTLASS backscatter along beam 5, and then deconvolving the shape of the radar pulse from the data, as illustrated in Fig. 3. The x-coordinate of each point represents the deconvolved width of the heated region across CUTLASS beams 3-7. This is determined by taking CUTLASS measurements along beams 3-7, at the range of the most intense scatter from the heated region. The width of the radar beam is then deconvolved from the data, as illustrated in Fig. 4. The dB points of the heater beam at $200 \mathrm{~km}$, calculated from the heater beam pattern depicted in Fig. 5, are also indicated. The $\mathrm{dB}$ points are different in azimuth and range, reflecting the asymmetrical pattern of the heater beam.

The Gaussian profiles fitted to the four O-mode heating periods between 1200 and 1240 on 26 April 1996 are depicted in Fig. 7. Figure $7 \mathrm{a}-\mathrm{d}$ illustrates the profiles fitted to the data taken across the beams, Fig. 7e to $h$ presents the profiles fitted to the data taken along beam 5 . The data are well represented by the Gaussian profiles, with the exception of the data in Fig. $7 \mathrm{f}$ and $\mathrm{h}$. These and other "bad fits", defined by a chi-squared fit less than 1, are excluded form the data plotted in Fig. 6.

An ionogram taken at the heater site at 1210 UT on 26 April 1996 is presented in Fig. 8. The dots represent the ionogram and the solid line indicates a calculated trueheight electron density profile (Titheridge, 1985). Figure 8 is typical of ionograms taken during the campaign.

\subsection{Estimating the size of the heated region}

The width of the deconvolved Gaussian provides a useful first estimate of the size of the heated region. This width represents approximately the $-2 \mathrm{~dB}$ contour of FAI amplitudes. However, it is also of interest to determine the limiting size of the region in which backscatter is generated above the background noise. This allows us to estimate the required heater threshold electric field for the excitation process. This may be achieved by exploiting the fitted Gaussian, as follows.

The returned backscatter power to the CUTLASS radar can be represented by

$P_{d B}=10 \log \left(\frac{S}{N}\right)$.

Where $P_{d B}$ is the returned backscatter power in $\mathrm{dB}$, and $S$ and $N$ are the signal and noise power respectively, measured in linear units. CUTLASS samples frequencies within a frequency band to obtain a quiet channel before transmitting. This sample determines the noise
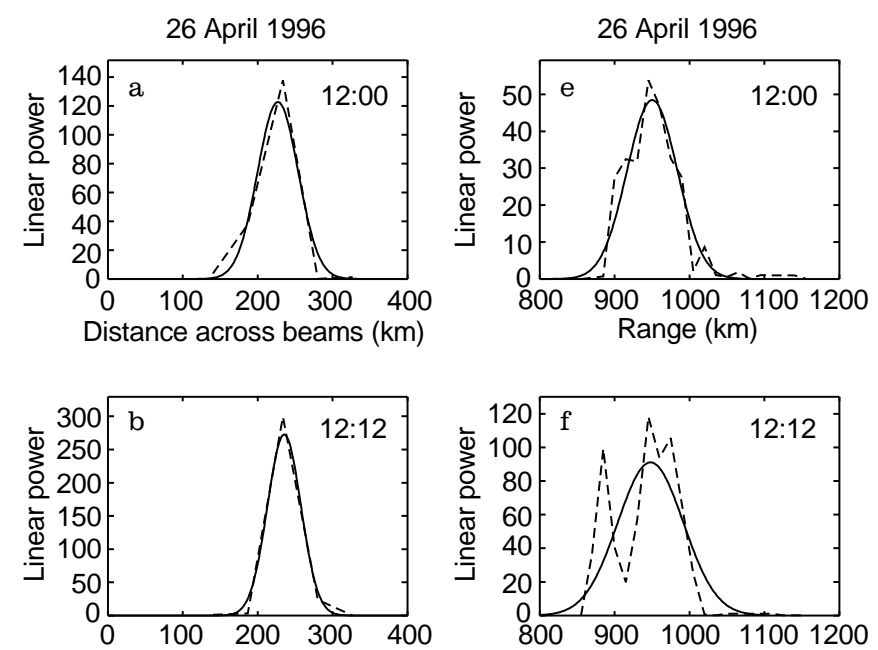

Distance across beams $(\mathrm{km})$
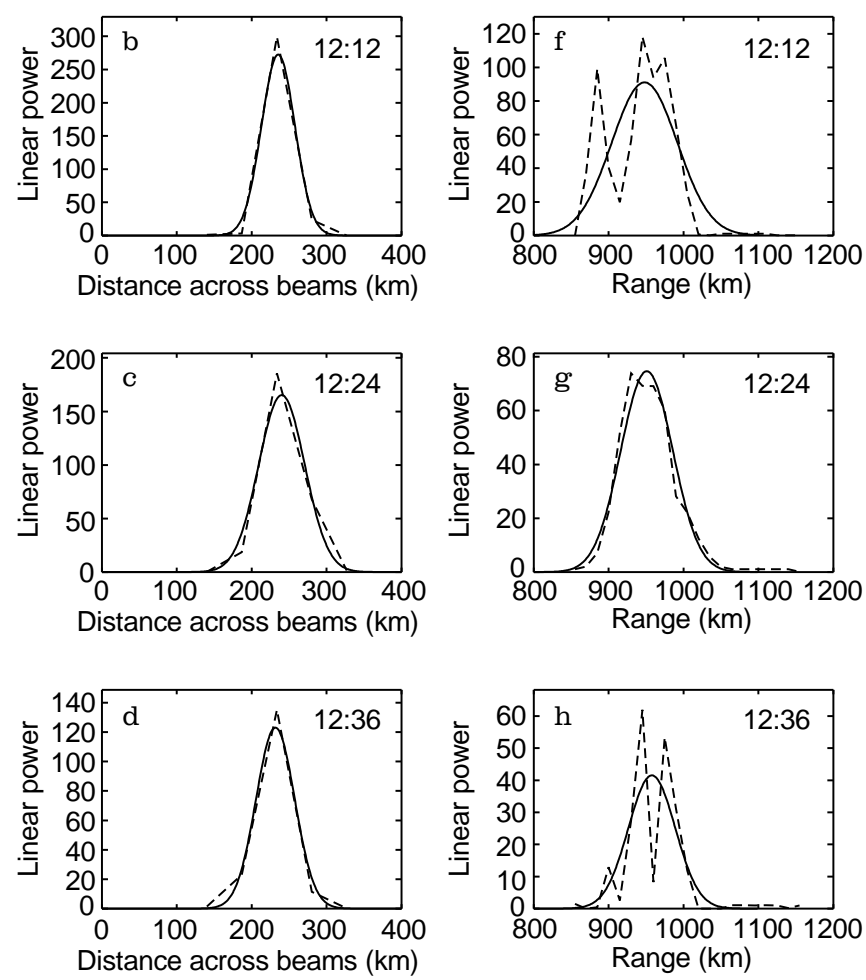

Fig. 7a-h. Data from CUTLASS Finland for 26 April 1996, between 1200 and 1240 UT. Panels a to d illustrate Gaussian profiles fitted to azimuthal data. Panels e to $\mathbf{h}$ present Gaussian profiles fitted to range data

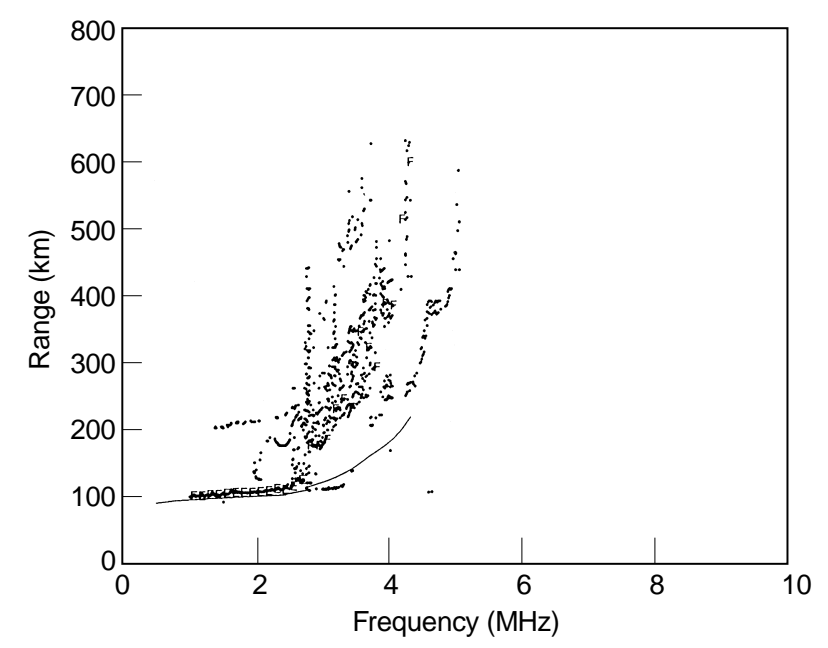

Fig. 8. An ionogram taken at 1210 UT on 26 April 1996. The critical frequency was $4.3 \mathrm{MHz}$. The solid line represents a fitted true-height electron density profile 
level, and at HF frequencies this is dominated by skynoise. From expression (4), the signal-to-noise ratio after deconvolution can be represented by a Gaussian of the form

$\frac{S}{N}=k_{\text {decon }} \mathrm{e}^{-x^{2} / 2 \sigma_{\text {decon }}^{2}}$,

where $\sigma_{\text {decon }}$ is the width of the Gaussian (equal to $\sqrt{\sigma_{\text {data }}^{2}-\sigma_{\text {pulse }}^{2}}$ as given in expression 4) and $k_{\text {decon }}$ is the peak height of the Gaussian. A value for $k_{\text {decon }}$ can be determined from the peak backscatter power from the centre of the heated region, $P_{\text {peak }}$. Substituting for $P_{\text {peak }}$, Eq. (6) becomes

$\frac{S}{N}=10^{\frac{P_{\text {peak }}}{10}} \mathrm{e}^{-x^{2} / 2 \sigma_{\text {decon }}^{2}}$

When the signal-to-noise ratio becomes unity, Eq. (7) can be re-written thus

$\frac{-P_{\text {peak }}}{10} \ln 10=\frac{-x^{2}}{2 \sigma_{\text {decon }}^{2}}$,

and the distance from the centre of the Gaussian where the signal reaches the noise level is

$x=\sqrt{\frac{\ln 10}{5} \sigma_{\text {decon }}^{2} P_{\text {peak }}}$.

From Eq. (9) we define the noise threshold range as the full range across the heated region where the deconvolved signal is above the noise level. This is equal to twice the value in Eq. (9).

noise threshold range $=1.36 \sigma_{\text {decon }} \sqrt{P_{\text {peak }}}$,

where the noise threshold range and $\sigma_{\text {decon }}$ are in $\mathrm{km}$ and $P_{\text {peak }}$ is in $\mathrm{dB}$.

Figure 9 presents observations of the noise threshold range as a function of UT for 22-26 April 1996. Each point represents backscatter measurements along CUTLASS beam 5, averaged over a $3 \mathrm{~min}$ O-mode heating period (X-mode periods did not produce significant scatter). Points are only plotted for an average peak returned power of greater than $20 \mathrm{~dB}$, and for a Gaussian profile fitting the data with a chi-squared fit less than 0.5. For the data in Fig. 9, the mean noise threshold range across the full width of the heated region is $170 \mathrm{~km} \pm 50 \mathrm{~km}$.
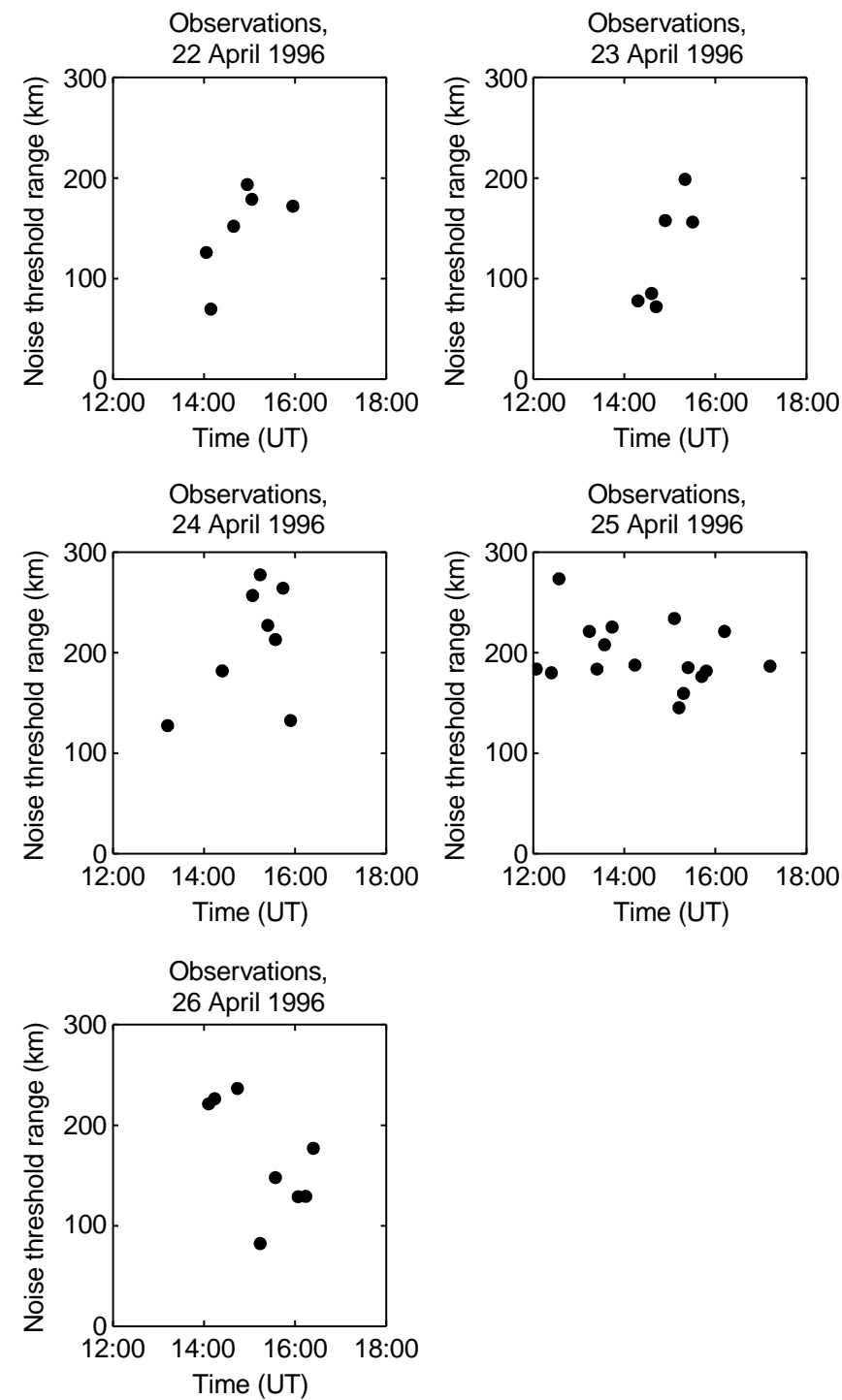

Fig. 9. Observations for 22-26 April 1996. The noise threshold range, a measure of where artificial scatter is observed above the noise level, plotted as a function of UT

By phasing the antennas in the heater array, it is possible to steer the heater beam to different positions. Figure 10 depicts three CUTLASS spatial plots of the heated region at $1447^{02}, 1504^{38}$ and $1533^{40}$ UT on 22

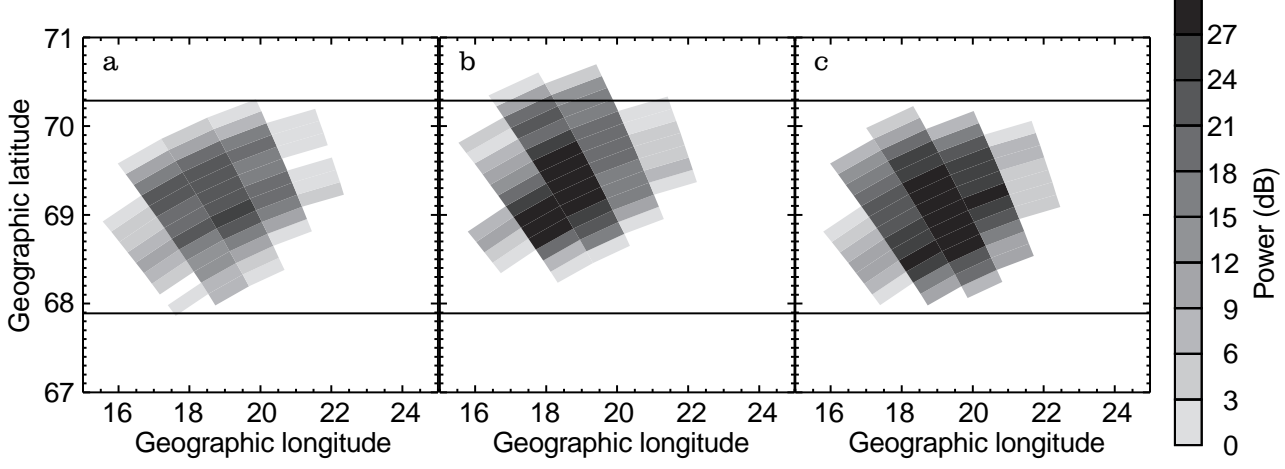

Fig. 10a-c. CUTLASS Finland spatial plots for 22 April 1996. a 1447 UT, heater beam at $-13^{\circ}$ to the vertical. b $1504 \mathrm{UT}$, heater beam pointing vertically. c $1534 \mathrm{UT}$, heater beam at $-13^{\circ}$ to the vertical. Horizontal lines are drawn at $67.9^{\circ}$ and $70.3^{\circ}$ latitude 
April 1996. During these three periods the heater was transmitting at $4.10 \mathrm{MHz}$ in O-mode polarisation. CUTLASS was in a 5 beam scan mode, dwelling on each beam for $5 \mathrm{~s}$ and transmitting at $12.4 \mathrm{MHz}$. Figure $10 \mathrm{a}$ and $\mathrm{c}$ represents data from when the heater beam pointed south, at $13^{\circ}$ to the vertical, Fig. $10 \mathrm{~b}$ represents data taken during vertical propagation. Horizontal lines are drawn at $67.9^{\circ}$ and $70.3^{\circ}$ latitude, to indicate clearly the movement of the heated region. The scatter corresponding to vertical propagation is $0.3^{\circ}$ north of the regions of scatter corresponding to propagation at $13^{\circ}$ to the vertical. No significant change is observed in the east-west position of the heated region.

Figure 11a depicts a spatial plot from the Iceland radar for $1324^{45} \mathrm{UT}$ on the 24 April 1996. The Iceland radar was operating at a frequency of $15 \mathrm{MHz}$ and was in a $45 \mathrm{~km}$ range mode. Figure $11 \mathrm{~b}$ presents the spatial plot from the Finland radar for $1324^{48} \mathrm{UT}$. The Finland radar was operating at $12.4 \mathrm{MHz}$ in a $15 \mathrm{~km}$ range mode. The heater was transmitting at $4.20 \mathrm{MHz}$ in $\mathrm{O}$ mode polarisation.

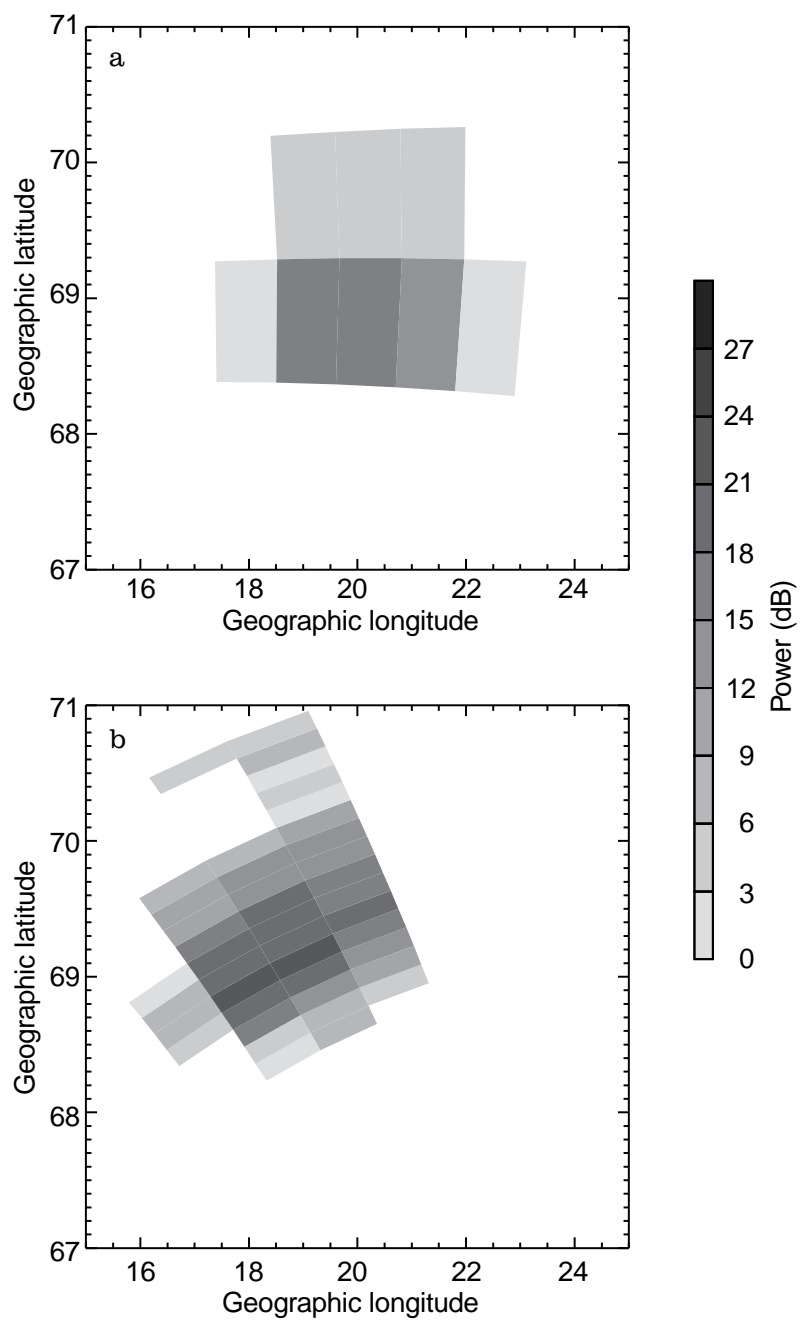

Fig. 11a, b. CUTLASS spatial plots of returned power taken at 1324 UT on 24 April 1996, during a heater-on period. a Iceland radar operating at $15 \mathrm{MHz}$ in $45 \mathrm{~km}$ range mode. b Finland radar operating at $12.4 \mathrm{MHz}$ in $15 \mathrm{~km}$ range mode

\section{Discussion and conclusions}

\subsection{CUTLASS spatial measurements}

The CUTLASS beam is approximately horizontal when it intersects the heated region, and so the data from CUTLASS represents a horizontal slice through the heated volume. The height at which CUTLASS intersects the heated volume encompasses the region of most intense scatter near the reflection point of the heater. The width of Gaussian functions fitted to the data indicate the variation in irregularity intensity with distance from the centre of the heated region, and allow comparison between measurements taken in range and azimuth. By determining where the Gaussian functions are above the noise level it is possible to make an estimate of the threshold electric fields required for the production of FAIs.

The heater transmitted a total of 212 heater-on periods during the April 1996 campaign, 187 in O-mode and 25 in X-mode. For each of these periods, the returned power detected by CUTLASS Finland along beam 5 (the beam directly over the heater site) was averaged for each range bin. Gaussian profiles were fitted to the resulting averaged data from each heateron, which could then be used in the deconvolution process. Of the 212 heater periods, Gaussian profiles fitted to 94 with a chi-squared fit of less than 1, indicating a reasonable approximation. Of the 118 periods which had a chi-squared fit greater than 1,93 had a peak returned power of less than $20 \mathrm{~dB}$. Scattered power during good experimental conditions was typically $30-40 \mathrm{~dB}$, and so data below $20 \mathrm{~dB}$ was deemed to represent poor conditions, and was disregarded. None of the $25 \mathrm{X}$-mode periods produced significant returned power, and this left $25 \mathrm{O}$-mode heater-ons with high returned power, but which did not have a Gaussian-like shape. These 25 heater-on periods were examined in more detail. Eight of the periods had a single-peaked form, but were not symmetrical enough to fit to a Gaussian. Three periods had a Gaussian-like shape, but did not quite meet the criteria of a chi-squared fit of less than 1. A further two profiles were dominated by natural scatter, and two periods indicated evidence of excitation outside the main heater beam. This left 10 periods which had a distinct double peaked shape. A possible explanation for this structure is that during heating at a frequency very close to the peak plasma frequency the vertically propagating heater ray penetrated the ionosphere, whilst rays propagating obliquely did not. This could also explain why many heater periods produced very low returned power, in some cases this could be because the plasma frequency was low enough for even the oblique waves to penetrate.

Figure 6 depicts data from the April 1996 campaign, during heater-on periods when CUTLASS was operating in a scan mode. The summary scatter plot for the whole campaign indicates that the deconvolved width of the heater beam corresponds approximately to the $2 \mathrm{~dB}$ point determined from the model. The width of the heater beam is usually described in terms of its half 
power point, or $3 \mathrm{~dB}$ down point, and so the Gaussian width of the heated region lies within the width of the heater beam.

The data in Fig. 7 represent range and azimuth profiles for 4 O-mode periods transmitted on 26 April 1996. The azimuthal data appears to be better represented by Gaussian profiles than the range data, as in nearly all cases the azimuthal data exhibited a singlepeaked, symmetrical shape. However, as only five beams were used in each scan, the number of degrees of freedom in azimuth is low, and the resulting chi-squared fit is poor. The limitations of the azimuthal data are borne out in Fig. 7. The azimuthal data is pictured in Fig. $7 \mathrm{a}-\mathrm{d}$ and the range data is in Fig. 7e-h. The range data depicts far more structure in the heated region. The data in Fig. $7 \mathrm{~g}$ is well represented by a Gaussian, and the data in Fig. 7e is also a reasonable fit. Figure $\mathrm{f}$ and $\mathrm{h}$ provides evidence of a double peaked structure. In contrast, the azimuthal data is all single-peaked, with no additional structure. This indicates that the finer resolution associated with the range data is crucial in revealing the true structure of the heated region. Although deconvolution of the azimuthal data gives an estimate of the spreading effect of the radar beam width, it cannot reveal any further detail when so few data points are available.

The threshold electric field required to produce irregularities can be estimated by utilising the mean noise threshold range determined from the data in Fig. 9, which has a value of $170 \pm 50 \mathrm{~km}$. The height where irregularities were excited was found to be around $200 \mathrm{~km}$ by observing the height of the ion-overshoot. The ion-overshoot is a spike in the power profile of the EISCAT data caused by the excitation of ion acoustic waves during the first few seconds of heating (e.g. Robinson, 1989). By using the beam pattern model presented in Fig. 5, the extent of $170 \mathrm{~km}$ was found to correspond to a heater power of $20 \pm 12 \mathrm{~dB}$ below the power at the centre of the beam. The large error estimate is due to the fast fall-off in heater power at this distance from the centre of the beam. During the experiments, six heater transmitters at a power of $90 \mathrm{~kW}$ each were utilised and the gain produced by the available antennas was $21 \mathrm{~dB}$, producing an ERP of $68 \mathrm{MW}$. Assuming free space propagation, the electric field, E $(\mathrm{V} / \mathrm{m})$, produced by a heater beam with $E R P$ in $\mathrm{kW}$, at an altitude, $R$ $(\mathrm{km})$, is (Robinson, 1989)

$E=\frac{0.25 \sqrt{E R P}}{R}$.

This gives an electric field of $0.3 \mathrm{~V} / \mathrm{m}$ at the centre of the heater beam at $200 \mathrm{~km}$ altitude. Allowing for the $20 \mathrm{~dB}$ reduction in power, the electric field at the edge of the region of artificial backscatter was between 0.01 and $0.1 \mathrm{~V} / \mathrm{m}$.

Hysteresis effects can significantly affect the threshold level of the irregularities. The heater operated in a typical cycle of 3 min O-mode on, followed by 3-min off. The irregularities were sometimes observed to remain in the ionosphere for as long as $3 \mathrm{~min}$ after the heater was off, but only at the centre of the heater beam and not at the edges where threshold effect were calculated. However, to be certain that hysteresis effect are not playing a role it is useful to examine the noise threshold range calculated for April 251996 (Fig. 9.). Between 1200 and 1500 UT the heater operated in a cycle of 2 min tune (low power on) $2 \mathrm{~min}$ off, $3 \mathrm{~min}$ O-mode, $3 \mathrm{~min}$ off. Between 1500 and 1700 UT the heater operated with a cycle of 3 min O-mode, 3 min off, 3 min X-mode, 3 min off. As X-mode heating does not produce irregularities, this means that between 1500 and $1700 \mathrm{UT}$ the ionosphere was left for $9 \mathrm{~min}$ before new irregularities were generated, compared to 2 min between 1200 and 1500 UT. The plot in Fig. 9. indicates that the noise threshold range remained at around $200 \mathrm{~km}$ for both heater regimes, indicating that hysteresis effects were not influencing the measurements.

The thermal parametric instability $(T P I)$ is thought to be involved in the production of FAIs, and this has a threshold electric field, $E_{t}$, given by (Das and Fejer, 1979)

$p E_{t}^{2}=\frac{15.38\left(T_{e o} k_{B}\right)^{2} \omega_{0}^{2} \sin I}{\nu \Omega_{e} \mathrm{e}^{2} H \lambda} \mathrm{e}^{\nu H / 50 \omega_{0}}$.

$p \leq 1$ depends on the standing wave ratio of the heater wave and is 1 for the case when the pump electric field is maximised, $k_{B}$ is Boltzmann's constant, $I$ is the angle of inclination of the geomagnetic field, $H$ is the scale height, $\omega_{0}$ is the angular frequency of the heater wave, $T_{\mathrm{eo}}$ is the undisturbed electron temperature, $v$ is the electron-neutral collision frequency, e is the electronic charge, $\lambda$ is the wavelength of the FAIs and $\Omega_{\mathrm{e}}$ is the electron gyro frequency. For typical ionospheric parameters Das and Fejer (1979) determine the following expression for $E_{t}$

$E_{t}=\sqrt{\frac{0.264}{\lambda p}}$.

For irregularities of scale length $10 \mathrm{~m}$, which is the case for a radar frequency of $15 \mathrm{MHz}$, and taking the most favourable case of $p=1$, the threshold electric field is $0.16 \mathrm{~V} / \mathrm{m}$. This threshold is high compared to the threshold value derived from the data of between 0.01 and $0.1 \mathrm{~V} / \mathrm{m}$, although other investigations have found a lower threshold value. Lee and Kuo (1983) consider a TPI characterised by a four-wave interaction. These authors found a threshold electric field of $0.0015 \mathrm{~V} / \mathrm{m}$ for irregularities scale lengths of greater than $10 \mathrm{~m}$, which is below the threshold electric-field measured for the current study.

These results indicate that it is possible to excite instabilities in a region significantly wider than the nominal width of the heater beam. Djuth et al. (1985) utilised a $49 \mathrm{MHz}$ coherent scatter radar to measure the response of the ionosphere to a heater wave reflecting in the E-region. These authors also observed scatter from FAIs outside the $3 \mathrm{~dB}$ point. Stubbe et al. (1982) found that under quiet ionospheric conditions, the level of anomalous absorption produced was independent of heater power. These authors observed $15 \mathrm{~dB}$ of anomalous absorption for a heater ERP of $160 \mathrm{MW}$. This did 
not change when the ERP was reduced by $10 \mathrm{~dB}$, which further illustrates that irregularities can be excited at relatively low heater powers.

Ray-tracing calculations (Jones and Stephenson, 1975) utilising the appropriate heater frequency and the ionogram in Fig. 8 suggest that rays spread out to a diameter $5 \mathrm{~km}$ greater than the nominal half-power point at the upper hybrid height. Other factors which could produce irregularities outside the $3 \mathrm{~dB}$ point are refraction due to natural horizontal gradients in the ionosphere and FAIs spreading outside the heated region along the field lines.

The heater antennas can be phased to provide electronic steering of the heater beam. In Fig. 10b the scatter, taken when the heater was pointing vertically, is approximately $0.3^{\circ}$ latitude further north than the scatter in Fig. 10a and c, where the heater was pointing south, at $13^{\circ}$ to the vertical. This corresponds to a horizontal distance of $40 \pm 10 \mathrm{~km}$. This is consistent with the theoretical value of $45 \mathrm{~km}$, obtained from the geometric movement produced at $200 \mathrm{~km}$ altitude when the beam is moved by $13^{\circ}$.

The first observations of the heated region by the CUTLASS Iceland radar were made during the April 1996 campaign. Figure 11 presents two spatial plots of the heated patch, Fig. 11a is produced from Iceland data, and Fig. $11 \mathrm{~b}$ presents data from the Finland radar. The different views of the heated region presented by the two plots are due to the position of the two radars in relation to the heater. The heater site at Tromsø is $1000 \mathrm{~km}$ away from the Finland radar and lies near the centre of the radar field of view. The Iceland radar is around $2000 \mathrm{~km}$ away from the Tromsø site and the heated region is only detected in the last two or three beams of the radar's scan (see Fig. 2). Also, in this case, the Finland radar was operating in a $15 \mathrm{~km}$ range mode, compared to the $45 \mathrm{~km}$ mode employed in Iceland. The low signal-to noise ratio observed at Iceland could be due to the greater range involved, and may also be due to greater refraction causing the radar beam to look into the E-region rather than the F-region over Tromsø. The Finland radar received backscatter from artificial irregularities consistently throughout the campaign. The results from Iceland were more sporadic, scatter was not detected during long periods of heating, but was sometimes observed consistently for periods of up to two hours.

The two spatial plots in Fig. 11 provide a consistent picture of the position of the heated region over the Tromsø site, at around $69^{\circ}$ latitude and $19^{\circ}$ longitude.

\section{Summary}

Measurements of the spatial extent of the heated region over Tromsø have been presented. The CUTLASS radar was employed to produce spatial maps of backscatter intensity across a horizontal cross section of the heated region. A deconvolution method, using fitted Gaussians, was utilised to remove the effects of the radar beam width and the pulse length from the measurements. The full Gaussian width of scatter from the heated patch was found to lie within the half-power-width of the heater beam. The profile of returned power along the radar beam was found to have a Gaussian-like shape in the majority of cases. In the examples where this was not the case, a double peak structure was sometimes seen. This structure could be due to vertically propagating heater rays penetrating the ionosphere, leaving only oblique rays to produce FAI. Measurements in azimuth produced Gaussian-like profiles in most cases, with no evidence of double peaked structure. This is probably due to the azimuthal data having poorer resolution than the range data, making this data suitable for estimating the overall width of the heated region, but not for identifying finer structure.

The horizontal range over which artificial instabilities were excited was measured to be around $170 \mathrm{~km}$. This demonstrates that it is possible to produce FAIs far beyond the half-power-point of the heater beam. By comparing the observations to a model of the heater radiated power, it was found that FAIs were generated at $20 \mathrm{~dB}$ down from the power at the centre of the heater beam. This corresponds to an electric field of between 0.01 and $0.1 \mathrm{~V} / \mathrm{m}$ at an altitude of $200 \mathrm{~km}$, which is an indication of the threshold electric field required to excite FAIs. The measured threshold value is in approximate agreement with the theoretical value required to excite the thermal parametric instability.

The effect of moving the heater beam has been investigated. It was found that when the heater beam was moved by $13^{\circ}$ from the vertical, the region of artificial backscatter observed by CUTLASS moved $0.3^{\circ}$ latitude south. By assuming that the scatter was from near the reflection point of the heater, it was calculated that the heated region had moved around $50 \mathrm{~km}$, which was consistent with a beam movement of $13^{\circ}$.

Spatial plots from the Finland and Iceland CUTLASS radars have been compared. The Finland radar produces more intense and more consistent scatter that Iceland, which is further away from the heater site and may not always look into the F-region. The two radars provide a consistent picture of the position of the heated region over Tromsø.

Acknowledgements. We thank Dr E. C. Thomas and his colleagues for operating CUTLASS and supplying the data. CUTLASS is funded by the Particle Physics and Astronomy Research Council (PPARC), with additional contributions from the scientific agencies of Sweden and Finland. Thanks are also due to the Director of EISCAT and his staff for operating the facility and supplying the data. EISCAT is an association of the national scientific agencies of Finland, France, Germany, Norway, Sweden, Japan and the United Kingdom. G. E. Bond is supported by a studentship from PPARC.

Topical Editor D. Alcaydé thanks P. A. Bernhardt and T. B. Leyser for their help in evaluating this paper.

\section{References}

Belenov. A. F., V. A. Bubnov, L. M. Erukhimov, Y. V. Kiselev, G. P. Komrakov, E. E. Mityakova, L. N. Rubstov, V. P. Uryadov, V. L. Frolov, Y. V. Chagnuov, and B. V. Ykhmatov, Parameters of 
artificial small-scale ionospheric irregularities, Radiophys. Quantum Electron. Engl. Transl., 20, 1240-1250, 1977.

Coster, A. J., F. T. Djuth, R. J. Jost, and W. E. Gordon, The temporal evolution of 3-m striations in the modified ionosphere, J. Geophys. Res., 90, 2807-2818, 1985.

Das, A. C., and J. A. Fejer, Resonance instability of small-scale field-aligned irregularities, J. Geophys. Res., 84, 6701-6704, 1979.

Djuth, F. T., R. J. Jost, S. T. Noble, W. E. Gordon, P. Stubbe, H. Kopka, E. Nielsen, R. Boström, H. Derblom, А̊. Hedberg, and B. Thidé, Observations of E region irregularities generated at auroral latitudes by a high-power radio wave, J. Geophys. Res., 90, 12 293-12 306, 1985.

Greenwald R. A., K. B. Baker, J. R. Dudeney, M. Pinnock, T. B. Jones, E. C. Thomas, J-P. Villain, J-C. Cerisier, C. Senior, C. Hanuise, R. D. Hunsucker, G. Sofko, J. Koehler, E. Nielsen, R. Pellinen, A. D. M. Walker, N. Sato, and H. Yamagishi, DARN/SuperDARN: a global view of the dynamics of highlatitude convection, Space Sci. Rev., 71, 761-796, 1995.

Hanuise C., A. Hedberg, J. Oksman, E. Nielsen, P. Stubbe and, H. Kopka, Comparison between the ionospheric plasma drift and the motion of artificially induced irregularities as observed by HF backscatter radars, Ann. Geophysicae, 4, A, 1, 49-54, 1986.

Hedberg, Å., H. Derblom, G. Wannberg, B. Thidé, H. Kopka, and P. Stubbe, Measurements of HF backscatter cross sections for striations created by ionospheric heating at different power levels, Radio Sci., 21, 117-125, 1986.

Hedberg, Å., H. Derblom, B. Thidé, H. Kopka, and P. Stubbe, Observations of HF backscatter associated with the heating experiment at Tromsø, Radio Sci., 18, 840-850, 1983.

Hibberd, F. H., E. Nielsen, P. Stubbe, H. Kopka, and M. T. Rietveld, Production of auroral zone E region irregularities by powerful HF heating, J. Geophys. Res., 88, 6347-6351, 1983.
Jones, R. M., and J. J. Stephenson, A versatile three-dimensional raytracing computer program for radio waves in the ionosphere, Techn Rep OT75-76, U.S. Department of Commerce, Washington D.C., USA, 1975.

Korovin, A. V., A. M. Nasyrov, E. V. Proskurin, and N. N. Yagnov, Doppler frequency variations in the field-aligned scattering of VHF waves by artificial irregularities of the ionosphere, Radiophys. Quantum Electron. (Engl. Transl.) 25, 195-199, 1982.

Lee, M. C., and S. P. Kuo, Excitation of upper-hybrid waves by a thermal parametric instability, J. Plasma Phys., 30, part 3, 463478, 1983.

Rietveld, M. T., H. Kohl, H. Kopka, and P. Stubbe, Introduction to ionospheric heating at Tromsø- 1 . Experimental overview, $J$. Atmos. Terr. Phys., 55, 577-599, 1993.

Rishbeth, H., and P. J. S. Williams, The EISCAT Ionospheric Radar: the system and its early results, Q.J. R. Astron. Soc., 26, 478-512, 1985.

Robinson, T. R., The heating of the high latitude ionosphere by high power radio waves, Phys. Rep., 179, 79-209, 1989.

Robinson, T. R., A. J. Stocker, G. E. Bond, P. Eglitis, D. M. Wright, and T. B. Jones, O- and X-mode heating effects observed simultaneously with the CUTLASS and EISCAT radars and low power HF diagnostics at Tromsø, Ann. Geophysicae, 15, 134-136, 1997.

Stubbe, P., H. Kopka, T. B. Jones, and T. R. Robinson, Wide band attenuation of radio waves caused by powerful HF waves: saturation and dependence on ionospheric variability, J. Geophys. Res., 87, 1551-1555, 1982.

Thome, G. D., and D. W. Blood, First observations of RF backscatter from field-aligned irregularities produced by ionospheric heating, Radio Sci., 9, 917-921, 1974.

Titheridge, J. E., Ionogram analysis - least-squares fitting of a Chapman-layer peak, Radio Sci., 20, 247-256, 1985. 